\title{
Time-Series Spectroscopy of the subdwarf B Star PG 1219+534
}

\author{
J. R. Eggen, ${ }^{1}$ M. D. Reed, ${ }^{1}$ S. J. O'Toole, ${ }^{2}$ J. H. Telting, ${ }^{3}$ R. $\varnothing$ stensen, ${ }^{4}$ D. M. Terndrup, ${ }^{5}$ \\ S. L. Harms, ${ }^{1}$ A.-Y. Zhou, ${ }^{1}$ R. L. VanWey ${ }^{1}$ \\ 1 Missouri State University, Missouri, USA \\ 2 Anglo-Australian Observatory, PO Box 296, Epping NSW 1710, Australia \\ ${ }^{3}$ Nordic Optical Telescope, Apartado 474, 38700 Santa Cruz de La Palma, Spain \\ ${ }^{4}$ Instituut voor Sterrenkunde, Katholieke Univ. Leuven, Celestijnenlaan 200 D, B-3001 Leuven, Belgium \\ ${ }^{5}$ Department of Astronomy, The Ohio State University, 140 W. 18th Avenue, Columbus, Ohio, USA
}

\begin{abstract}
We report on the progress of the analysis of our time-series spectroscopy of the pulsating sdB star PG 1219+534. PG 1219+534 has four stable pulsation frequencies $(6721,6961$, 7490 , and $7807 \mu \mathrm{Hz}$ ) with nearly constant photometric amplitudes. The pulsation spectrum is simple enough to be resolved within a couple of hours, yet complex enough that multiple $\ell$-degrees must be present. Our data set consists of 5072 spectra obtained in April 2004 from the Mayall $4 \mathrm{~m}$ at Kitt Peak National Observatory (KPNO), at $1.4 \AA \AA$ pixel resolution, and the Nordic Optical Telescope (NOT), with $0.8 \AA$ /pixel resolution. Though still under analysis, we have already measured line-centroid variations to discern pulsation velocities using Balmer and $\mathrm{He} \mathrm{I}$ lines between 3750 and $5000 \AA \AA$.
\end{abstract}

\section{Preliminary Results}

We have detected significant radial velocity variations from the NOT data with marginal detections in the KPNO data. The left panels of Fig. 1 show the Fourier transforms of the KPNO, NOT, and total data sets. Insets are the data windows, solid lines indicate the $4 \sigma$ detection limit and dashed lines indicate the positions of the photometric pulsations. We have noticed substantial differences in velocity amplitudes between the KPNO and NOT data, which are temporally separated by one week. Photometric amplitudes, obtained simultaneous to the spectroscopic data are stable. Night-to-night velocity variations are also marginally detected in the NOT data. We suspect these may be a systematic effect, but have not yet found the culprit. The NOT data were also phase-binned (Fig. 1, right panels) by the four known pulsation frequencies. While the scatter of the individual points is relatively large, average values (fit with a solid line) clearly show the velocity variations. We have also completed a preliminary search for equivalent width variations in the Balmer lines, but no variations were detected.

We are now completing a more thorough analysis of pulsation velocities, searching again for equivalent width variations, and fitting phase-binned spectra to atmospheric models to measure temperature, gravity, and changes in the helium ionization fraction caused by pulsation. Along with a velocity/photometry comparison, we anticipate that our results will constrain the modes of pulsation.

Acknowledgments. Support for MDR is from the National Science Foundation under Grant Number AST007480, the American Astronomical Society and Missouri State University. Travel for JRE was supported by the Missouri Space Grant Consortium. 

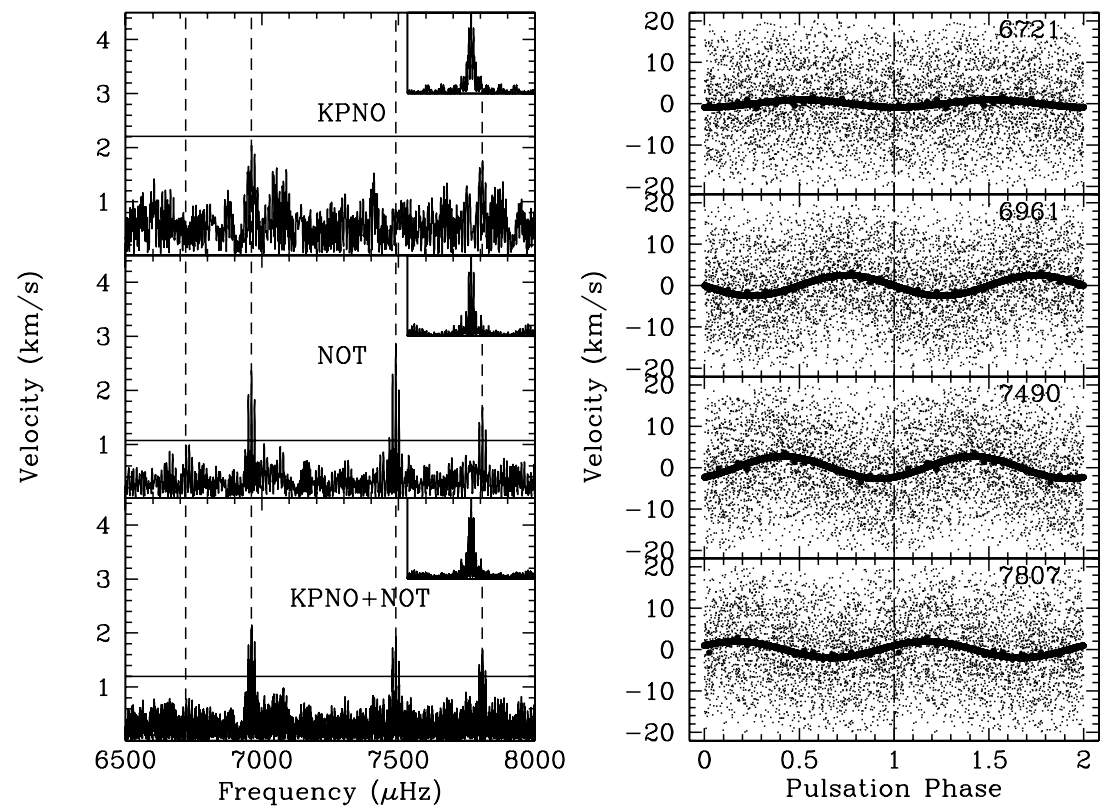

Figure 1: Left: Fourier transforms of radial velocity data. Right: Pulsation phase-binned velocities of the NOT data. The pulsation frequencies (in $\mu \mathrm{Hz}$ ) are provided in each panel along with a best-fit line. 